\title{
Development of Welding Multi-Information Remote Wireless Monitoring System Based on STM32
}

\author{
Haobo Liu, Jianfeng Yue, Wenji Liu, Haihua Liu, Liangyu Li \\ School of Mechanical Engineering, Tianjin Polytechnic University, Tianjin, China \\ Email: lhb142703@163.com
}

How to cite this paper: Liu, H.B., Yue, J.F., Liu, W.J., Liu, H.H. and Li, L.Y. (2020) Development of Welding Multi-Information Remote Wireless Monitoring System Based on STM32. Journal of Computer and Communications, 8, 29-39.

https://doi.org/10.4236/jcc.2020.812003

Received: November 6, 2020

Accepted: December 7, 2020

Published: December 10, 2020

Copyright $\odot 2020$ by author(s) and Scientific Research Publishing Inc. This work is licensed under the Creative Commons Attribution International License (CC BY 4.0).

http://creativecommons.org/licenses/by/4.0/

\begin{abstract}
A single sensor is used to obtain welding information in welding monitoring process, but this method has some shortcomings. In order to obtain more comprehensive and reliable welding information, this paper designed and built a welding multi-information wireless monitoring system with STM32F407ZET6 as the control core and ALK8266 as the wireless transmission module. Real-time acquisition, transmission and display of electric arc signal and welding image information are realized in the monitoring system. This paper mainly introduces the hardware and software core of the monitoring system. At the same time, the signal collected by the monitoring system is compared with the original signal, and the accuracy of the remote monitoring system is tested. The monitoring system is used in welding test. The test results show that the accuracy of the monitoring system meets the requirements, and the on-line monitoring of electric arc signal and welding image can be realized in the welding process.
\end{abstract}

\section{Keywords}

Electric Arc Signal, Welding Image, Wireless Monitoring, STM32, ALK8266

\section{Introduction}

With the development of welding automation, it becomes more and more important to monitor the welding process on-line effectively. Among them, the on-line monitoring of the welding of information acquisition is the most critical step, but the information obtained by a single sensor has shortcomings in terms of comprehensiveness and reliability, such as using hall current electrical signal can obtain in the process of welding arc voltage and the arc electrical signal ob- 
tained but susceptible to the influence of the high frequency magnetic field; The camera can be used to obtain welding image information, such as weld pool image and arc shape in the welding process, etc. However, these welding images are susceptible to arc interference, which may cause the inaccurate information obtained [1]. The traditional welding process monitoring system only collects and analyzes the electric arc signal, which has poor reliability and stability. Considering this problem, this paper designs a remote monitoring system that can simultaneously collect electric arc signal and welding image information in the welding process [2] [3], making full use of the different types of redundancy and complementarity between sensors. The monitoring system collects welding information from different angles, thus achieving more comprehensive and reliable monitoring of the welding dynamic process [4] [5].

At the same time, considering that due to the welding field industrial equipment, personnel and the actual situation with big liquidity caused by the traditional wired monitoring wiring difficulties, low extensibility, communication distance is restricted, increased costs and other issues, the monitoring system designed in this paper adopts ALK_8266 wireless transmission module, connects the network through the router node, and uses modBUS-TCP communication protocol for the communication between the remote acquisition terminal and the remote monitoring terminal [6] [7]. It is flexible in networking and has achieved good results in practical application [8] [9] [10].

\section{Overall System Design}

The overall design of the system is mainly divided into two parts: remote monitoring terminal and remote acquisition terminal. Each remote acquisition terminal is composed of welding machine, welding parts, Hall current and voltage sensor, camera and wireless transmission control module with STM32 as the core, which is mainly responsible for real-time acquisition, storage and transmission of welding arc electrical signals and image information. Each remote monitoring terminal is composed of a wireless receiving control module with STM32 as the core and an upper computer display module, which is mainly responsible for receiving and analyzing the welding information sent by the remote acquisition terminal and displaying and storing it on the upper computer interface. The wireless module ALK8266 is used to connect the remote acquisition terminal and the remote monitoring terminal to communicate through the router node, and the welding information collected by the acquisition terminal can be displayed on the display interface of the monitoring terminal in real time. The overall design drawing of the system is shown in Figure 1 below.

\section{Terminal Hardware Circuit Design}

\subsection{Overall Hardware Circuit Design}

The overall design of the monitoring system control module is shown in Figure

2 , which is mainly divided into four parts: data acquisition, data processing, data 


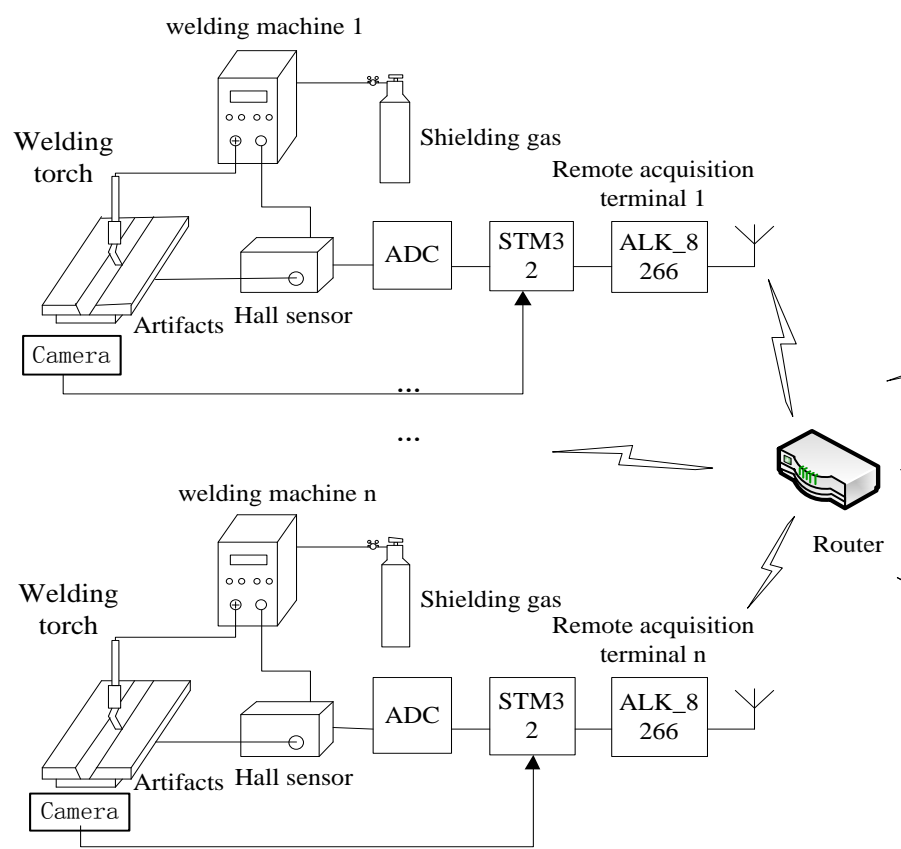

Remote acquisition terminal
Remote monitoring terminal 1
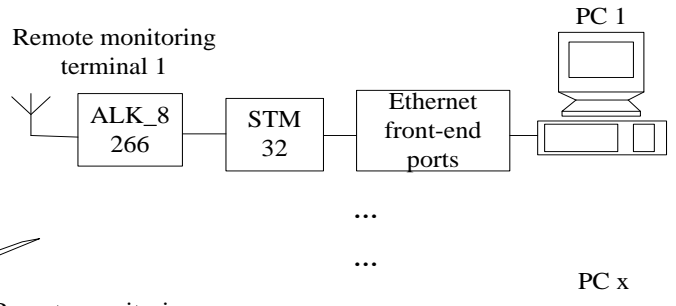

Remote monitoring

terminal $\mathrm{x}$
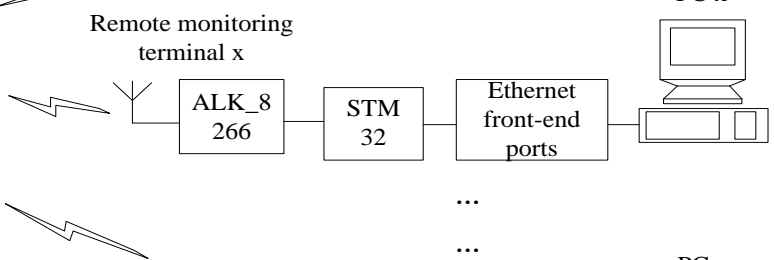

$\cdots$

Remote monitoring terminal $n$
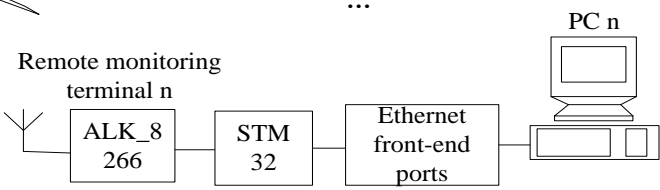

Remote monitoring terminal

Figure 1. Overall design of the system.
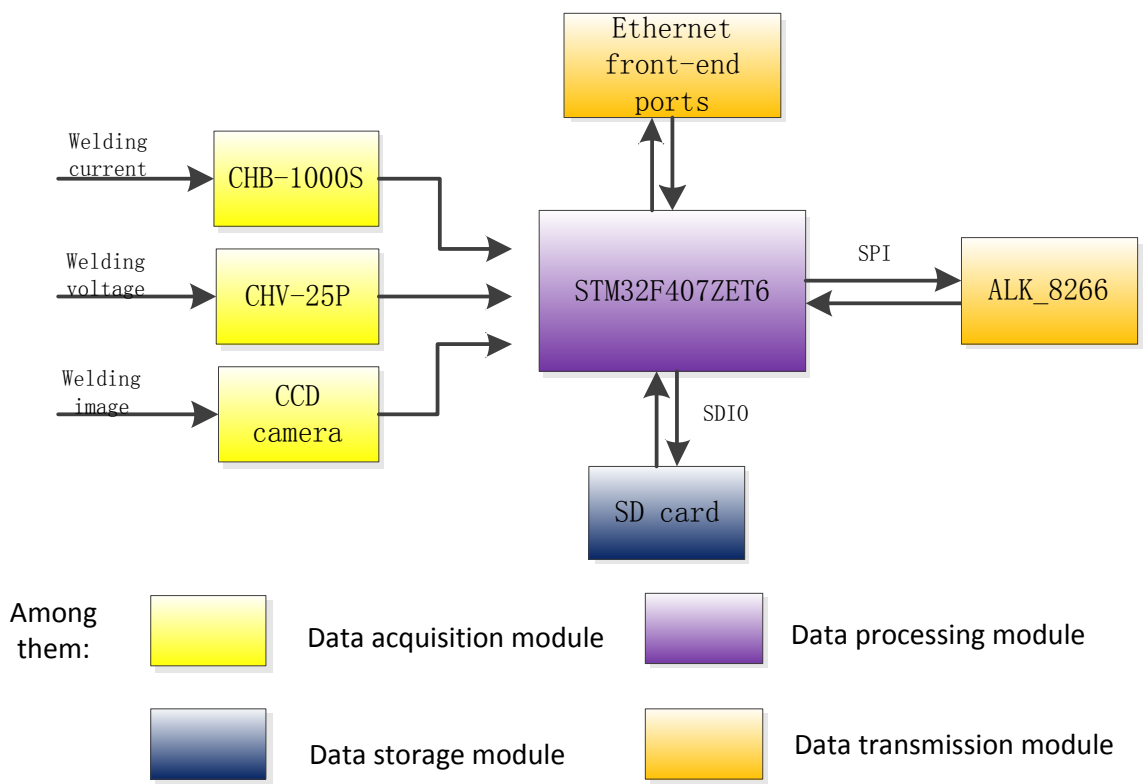

Figure 2. Overall hardware design.

storage and data transmission. The data acquisition module is divided into two parts, one of which is to collect electric arc signals through STM32's built-in $\mathrm{ADC}$, and the other is to collect welding image signals. In the data processing part, the collected data is encapsulated by Modbus_TCP data frame by the remote acquisition terminal, and then it is used as the response instruction to send to the remote monitoring terminal. After receiving the response instruction, the remote monitoring terminal parses Modbus_TCP data frames and displays them 
on the upper computer interface. The data storage part uses the SDIO interface to connect with the MCU, which is mainly used for data storage after the upper computer is disconnected. The data transmission part uses the wireless module to connect the peripheral equipment and MCU to the wireless network for the communication between the remote acquisition terminal and the remote monitoring terminal.

\subsection{Core Hardware Circuit Design}

The hardware core circuit is mainly divided into two parts: data acquisition and data transmission. Among them, welding current and voltage acquisition is carried out through the ADC provided by $\mathrm{MCU}$, and the hardware circuit design of welding image acquisition and data transmission is mainly introduced here.

\subsubsection{Hardware Circuit Design of Camera Interface}

The STM32F407ZET6 digital camera interface is used for image acquisition in this paper, and its external interface is shown in Figure 3 below:

Including DCMI_XCLK for provide the camera's sensor chip clock operation, DCMI_SCL and DCMI_SDA respectively used as written to the camera registers configuration parameters of temporal communication clock signal and time-series data signals, DCMI_PCLK, DCMI_VSYNC and DCMI_HREF respectively the pixel output clock, the camera pixels pixel row synchronization and frame synchronization signals, is used to control the camera pixel synchronization output. The channel DCMI_D0-DCMI_D7 is an 8-bit parallel interface for the camera data output, which is used to output the image signals collected by the camera to the outside. Through these interfaces, the camera is connected with the MCU, which realizes the control and communication of the CAMERA by the MCU.

\subsubsection{Hardware Circuit Design of Wireless Modules}

ALK_8266 wireless module is used for data transmission in this paper, and its reserved interface is shown in Figure 4. ALK8266 wireless module is powered

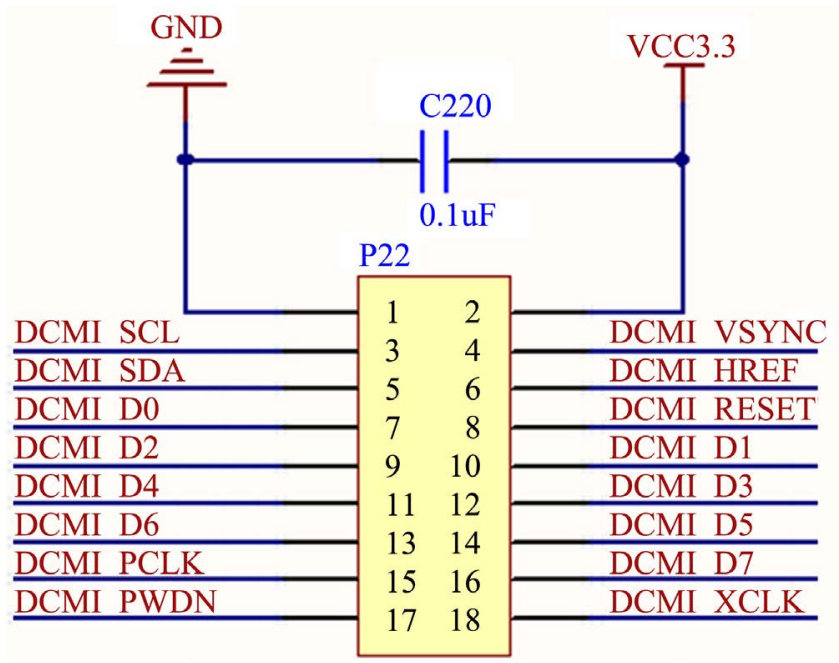

Figure 3. Camera interface. 


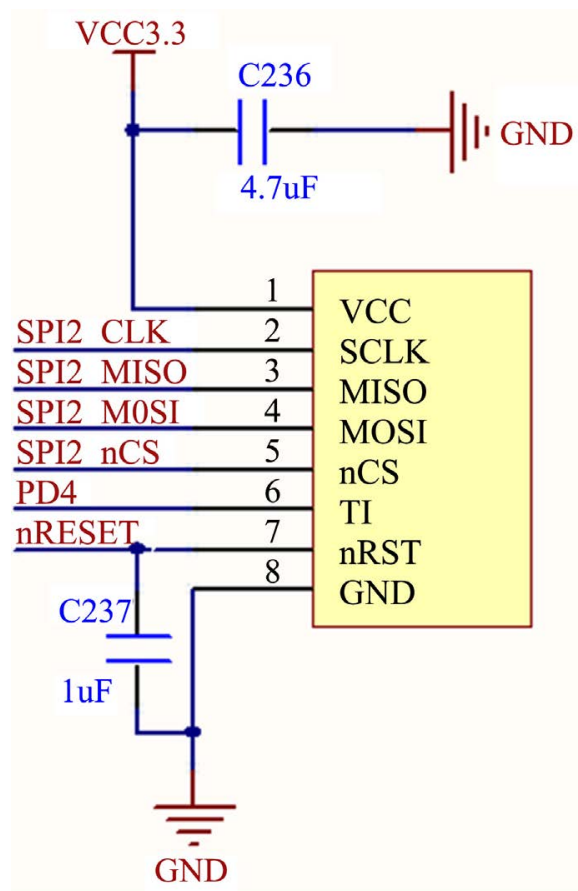

Figure 4. Interface diagram of ALK_8266 wireless module.

by $3.3 \mathrm{~V}$ voltage and communicates with MCU through SPI interface. The LED light on the module can show whether the module is working normally. The nRESET pin connected to the module is connected to the external key to achieve the module reset. When the key is pressed down, the module reset and returns to the initial state. External WIFI signals can be accessed again, and web page configuration Settings can also be carried out. The PD4 pin connected to the module $\mathrm{TI}$ is the module receiving interrupt to determine the pin. When the module receives data, the pin will output an ascending edge, which can be used as the external pin interrupt trigger source to determine whether the module has received data.

\section{Terminal Software Programming}

\subsection{Overall Software Programming}

Software program design in general can be divided into remote monitoring terminal and remote acquisition terminal programming, in which remote monitoring terminal program main function is to send remote terminal of the different types of polling instruction, thus receives the remote acquisition terminal to the corresponding response data frames, polling instructions in the data frame after parsing the data displayed on the PC screen. The main function of the remote acquisition terminal is to collect multi-sensor information, write the collected information into the response data frames of MODBUS-TCP, wait for the receiving of polling instructions from the remote monitoring terminal, and send the response data frames containing the collected data to the remote monitoring terminal. Its flow chart is shown in Figure 5 below. 


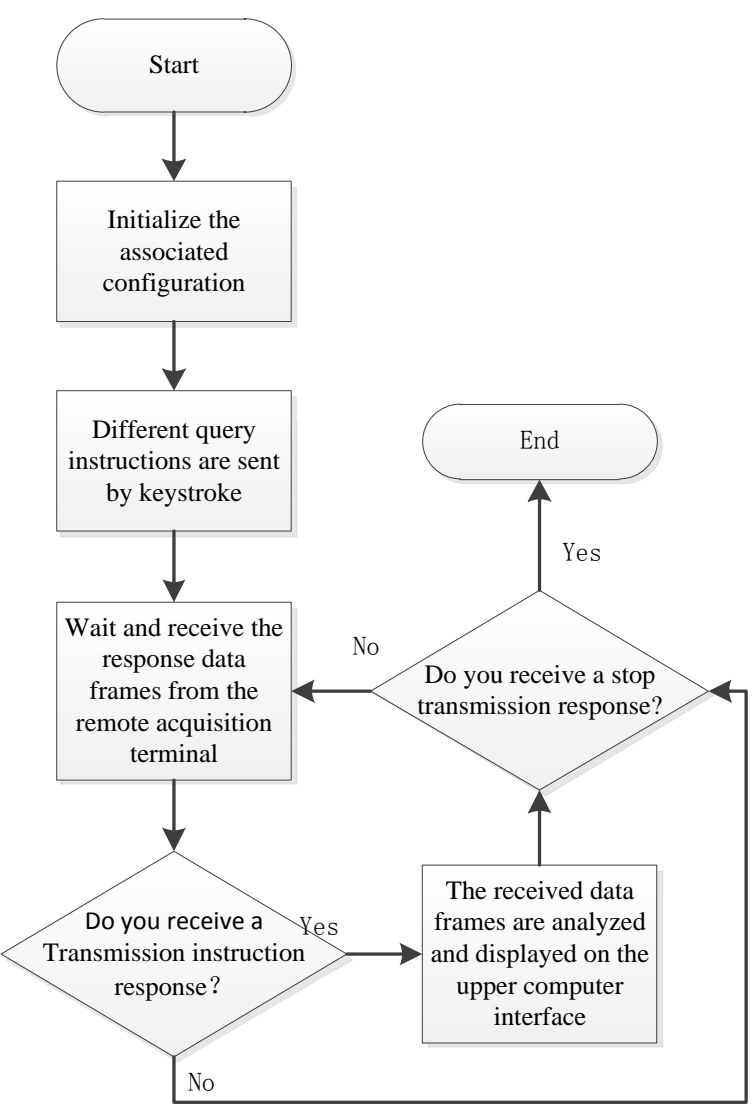

(a)

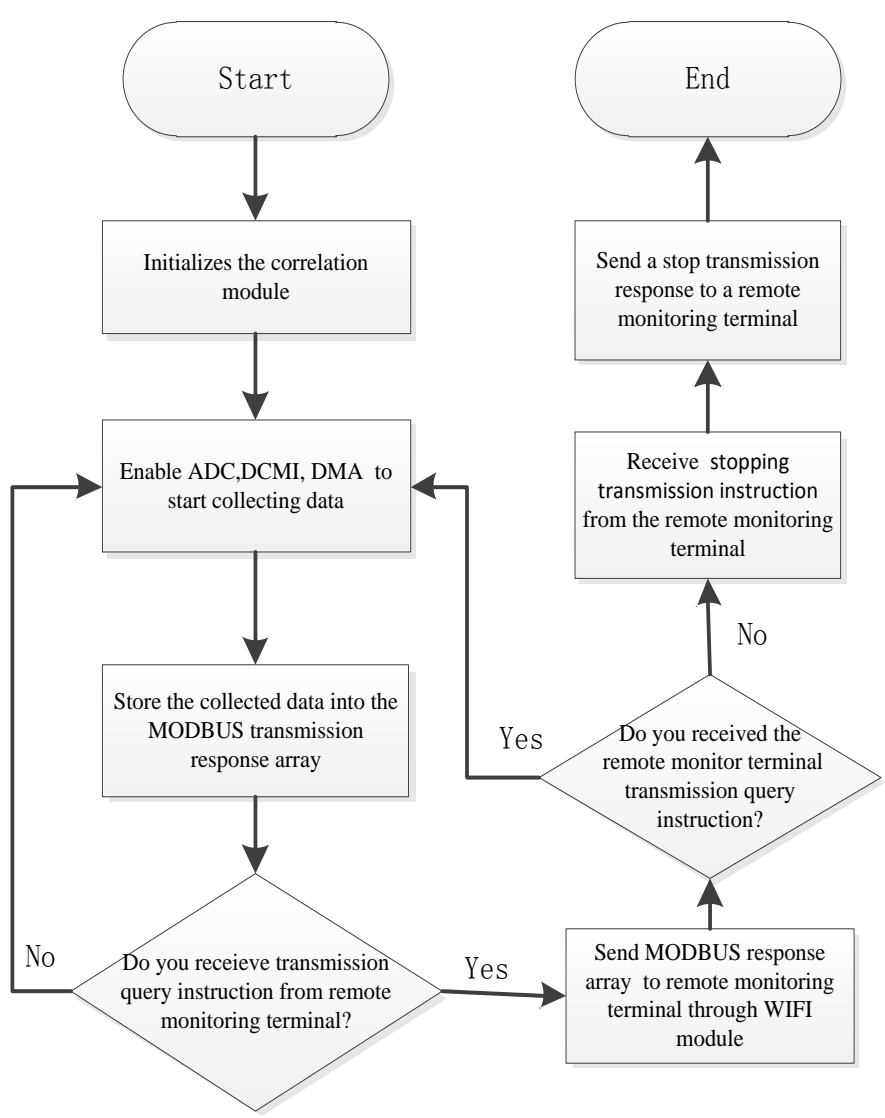

(b)

Figure 5. Overall program flow chart of the software. (a) Flow chart of remote monitoring terminal procedures; (b) Program flow chart of remote acquisition terminal.

\subsection{Core Software Programming}

The core part of the software is the acquisition of electric arc signal and image data. Its acquisition flow chart is shown in Figure 6 below. Among them, the current and voltage of the remote acquisition terminal is collected by the ADC embedded in the STM32 chip. The mean value is calculated once for every 500 times of collection, and then converted into the actual current and voltage value and stored in the modBUS-TCP data frame. Images are collected in DCMI interrupts. After each frame of image is collected, images are segmented into modbus-TCP data frames and wait for receiving polling instructions from remote monitoring terminals. After receiving the query instruction from the remote monitoring terminal, modBUS-TCP data frames are sent to the upper computer.

\subsection{ModBUS-TCP Communication Protocol}

The upper computer and the lower computer communicate through MODBUSTCP. When the upper computer sends query instructions to the lower computer, the lower computer sends corresponding response instructions to the upper computer. The sending query and response process of MODBUS-TCP is shown in Figure 7 below. 


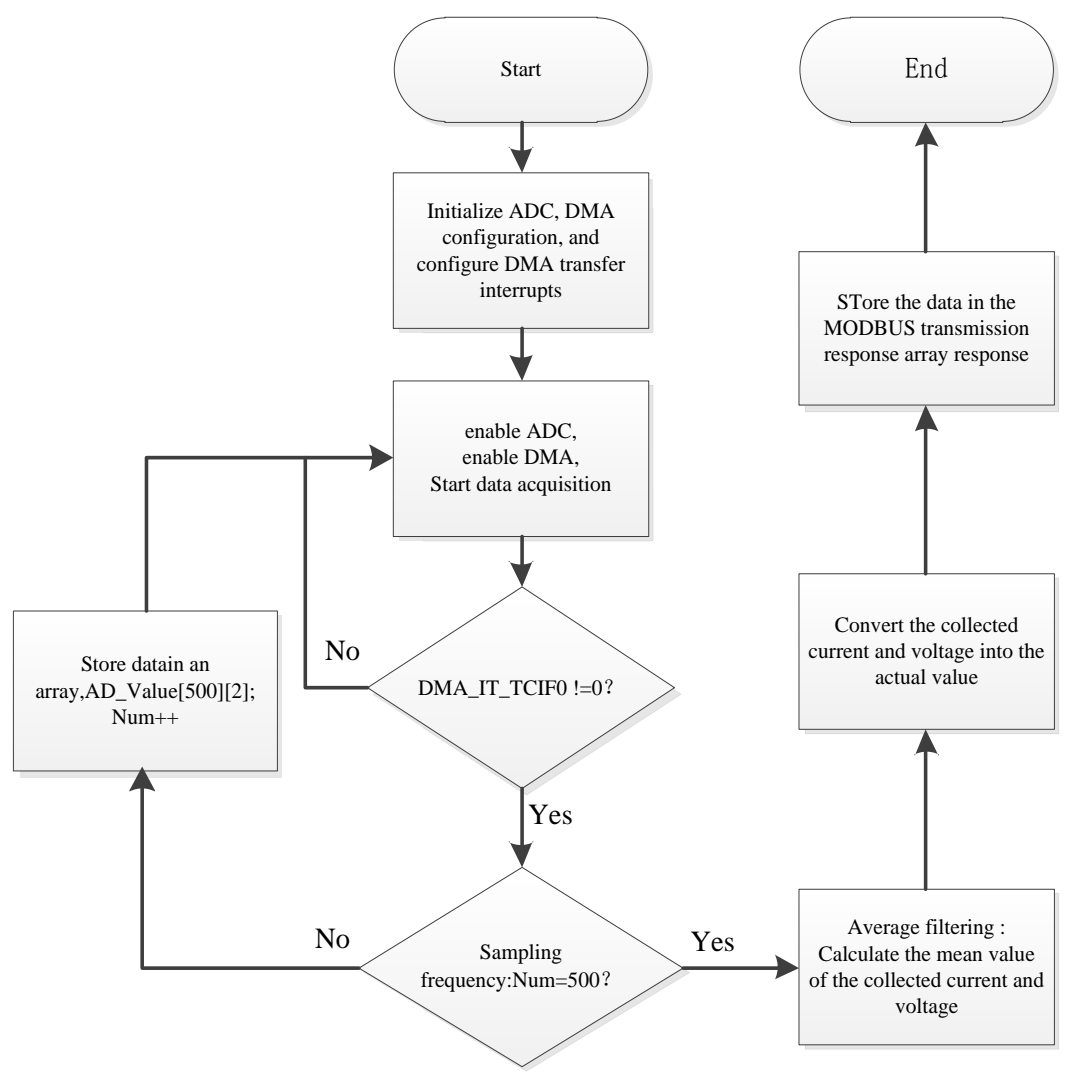

(a)

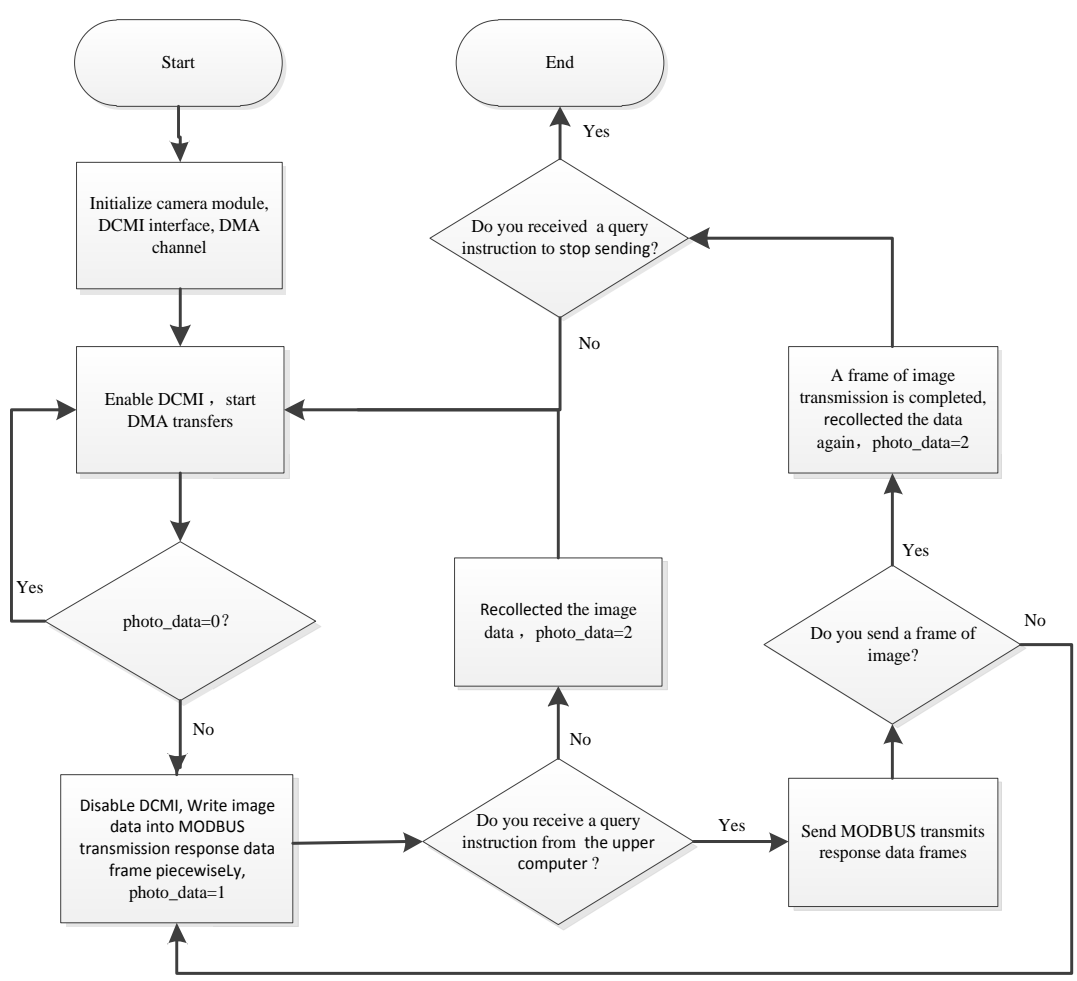

(b)

Figure 6. Welding data acquisition flow chart. (a) Flow chart of welding current and voltage data acquisition; (b) Welding image acquisition flow chart. 


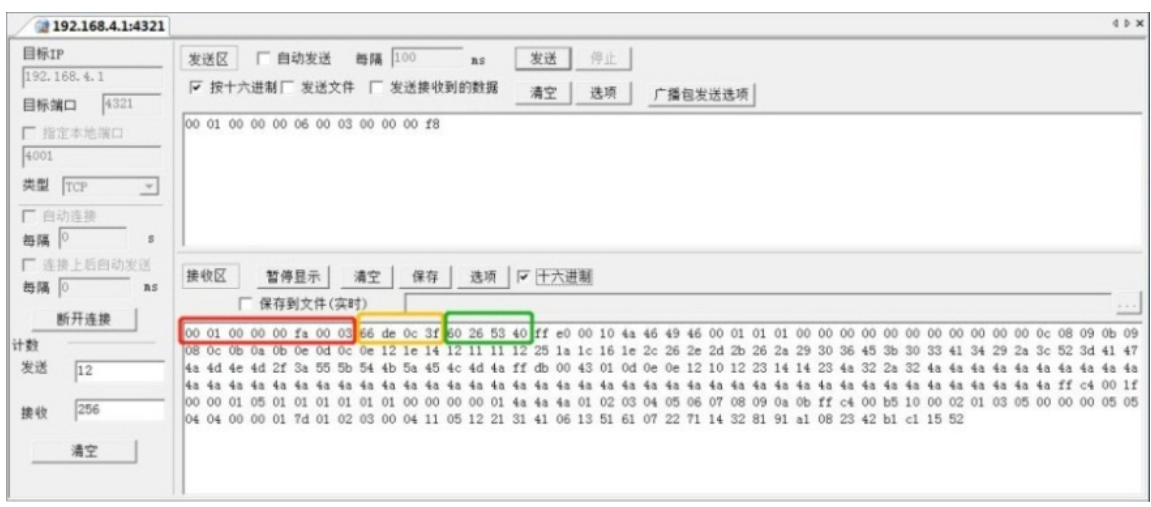

Figure 7. MODBUS-TCP sending query response process.

The sending query instruction of MODbus-TCP is: 0001000000060003000000 f8 (12 bytes).

The sending response instruction of MODBUS-TCP is:

0001000000 fa 0003 Current1 (4 bytes) Voltagel (4 bytes) Imagel (24 bytes).

\section{Accuracy Detection and Test Analysis of Monitoring System}

\subsection{Accuracy Detection of Monitoring System}

In this paper, the welding image transmission speed is up to $200 \mathrm{Mbps} / \mathrm{s}$, and the transmitted image is clear and reliable, which can be used for monitoring and processing. The accuracy detection here is mainly to test and verify the accuracy of the current and voltage signals received at the upper computer end. The 0 $3.3 \mathrm{~V}$ voltage generated by the function generator is collected by the remote monitoring system. Every 500 points are collected for mean processing, and the relative error between the signals at the receiving end and the input end is compared with the actual input voltage. The results are shown in Table 1 below:

The test results show that the error between the voltage value collected by the monitoring system and the actual input voltage value is basically within $1 \%$, and the accuracy meets the requirements, which can realize the remote real-time monitoring of arc signal in the dynamic welding process.

\subsection{Multi-Information Monitoring Test and Analysis of Welding Process}

The monitoring system designed in this paper was applied in the welding test, which was operated by The Forness welder. Among the welding parameters, the wire feeding speed was $5 \mathrm{Vf} /\left(\mathrm{m} \cdot \mathrm{min}^{-1}\right)$, the welding material was Q235, the wire diameter was $1 \mathrm{~mm}$, the protective gas was $80 \% \mathrm{CO}_{2}$ and $20 \% \mathrm{Ar}$, the welding speed was $5 \mathrm{~mm} \cdot \mathrm{S}^{-1}$, the swing speed was $10 \mathrm{~mm} \cdot \mathrm{S}^{-1}$, and the swing size was 3 $\mathrm{mm}$.

During the welding process, the upper computer interface prepared by LabView on the remote monitoring terminal is observed to have a real-time understanding of the changes in the welding process, as shown in Figure 8 below, the 
Table 1. Arc acquisition precision table.

\begin{tabular}{ccc}
\hline $\begin{array}{c}\text { Actual input voltage } \\
\mathrm{U} / \mathrm{V}\end{array}$ & $\begin{array}{c}\text { Collect voltage } \\
\mathrm{U} / \mathrm{V}\end{array}$ & $\begin{array}{c}\text { The relative error } \\
\%\end{array}$ \\
\hline 1 & 0.996741 & -0.325 \\
1.5 & 1.506524 & 0.652 \\
2 & 2.005145 & 0.514 \\
2.5 & 2.495356 & 0.464 \\
3 & 3.005461 & 0.546 \\
3.3 & 3.295166 & 0.483 \\
\hline
\end{tabular}

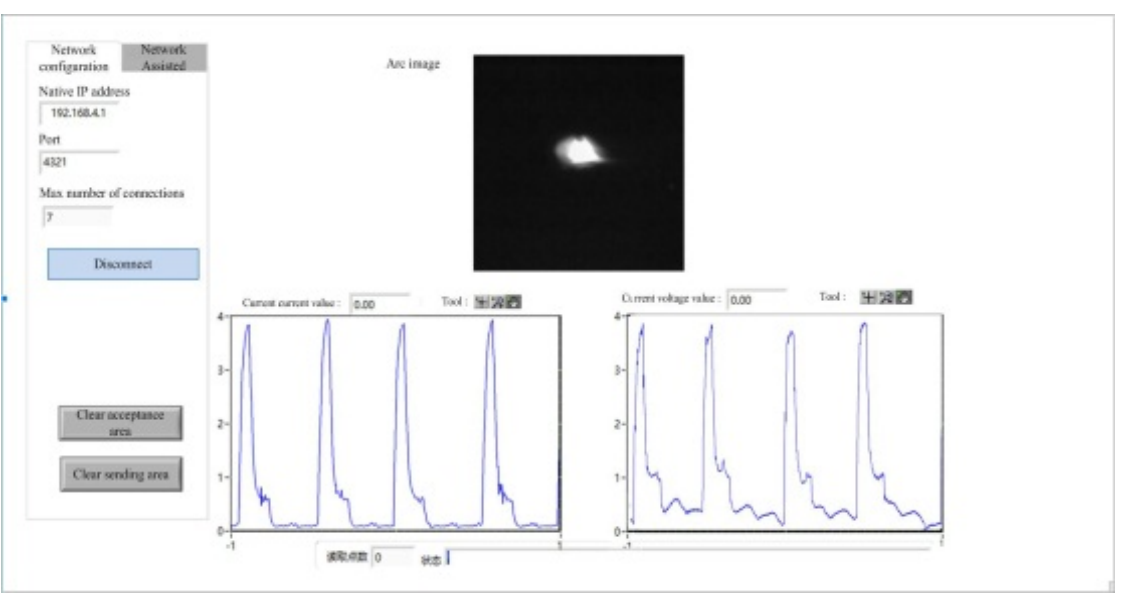

(a)

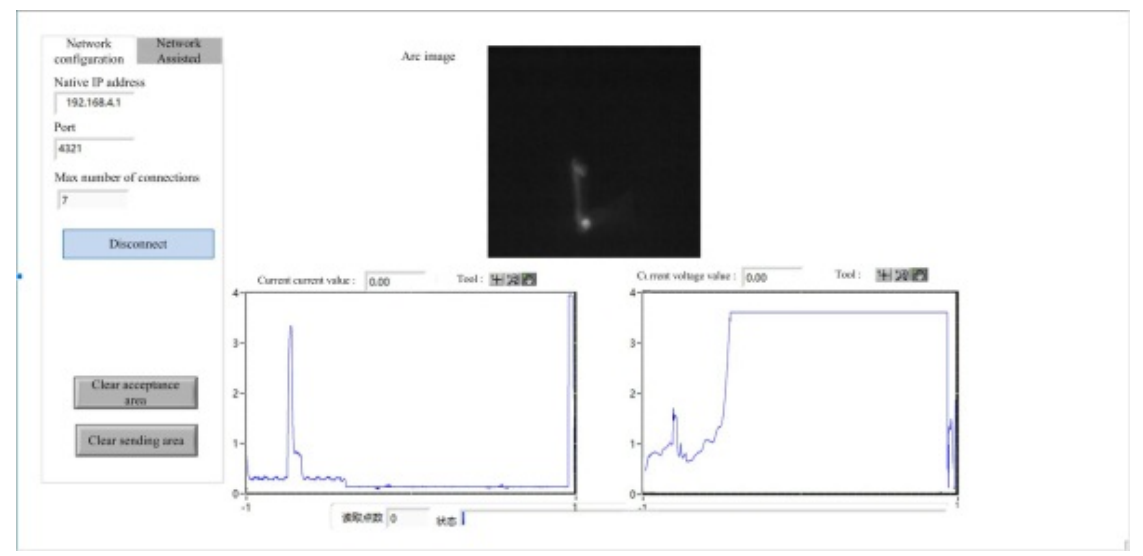

(b)

Figure 8. Real-time monitoring interface of welding process. (a) The monitoring interface during the normal welding process; (b) Monitoring interface in case of arc break.

change process of welding arc electrical signal and image information in the welding process can be observed in a real-time and intuitive manner from the upper computer interface, Figure 8(a) shows the electric arc signal and image signal during normal welding. When the welding state changes, the signal at the monitoring interface also changes, as shown in Figure 8(b) are breaking occur at this time, current voltage waveform changes. 


\section{Conclusion}

In this paper, a set of STM32F407ZET6 based multi-information remote wireless monitoring system for welding process is designed and built. Through real-time collection, processing, transmission and display of welding arc signal and welding image signal in the welding process, multi-information remote online monitoring of welding dynamic process is realized. The welding on-line monitoring system is stable and reliable, which provides a good source of information for follow-up welding seam tracking and quality monitoring. However, this paper only collects electric arc signal and welding image information, and does not collect some information that can represent the internal condition of welding seam, such as sound signal. On the basis of the monitoring system established in this article, the multi-information monitoring system still has a lot of room for development.

\section{Acknowledgements}

This paper is under the project of Tianjin Enterprise Science and Technology Commissioner (Project Number: 20YDTPJC01060).

\section{Conflicts of Interest}

The authors declare no conflicts of interest regarding the publication of this paper.

\section{References}

[1] Zhang, Z.F. (2015) Study on Welding Defect Feature Extraction of Aluminum Alloy Pulse GTAW Process Based on Multi-information Fusion. Shanghai Jiao Tong University, Shanghai.

[2] Yan, P.Y., Duan, R.B. and Liu, W.J. (2018) Design of High Precision Data Acquisition System for Welding Arc Electric Signal Based on AD7606. Hot Working Process, 47, 176-179.

[3] Yu, H. (2016) Design and Implementation of Remote Distributed Programmable Automatic Control System Based on STM32. Zhejiang University, Hangzhou.

[4] Wang, Y.N. and Li, S.T. (2001) Overview of Multi-Sensor Information Fusion and its Application. Control and Decision Making, 16, 518-522.

[5] Ma, P. (2006) Basic Principle and Application of Multi-sensor Information Fusion. Control Engineering, 13, 5.

[6] Zhang, Z.H. (2015) Implementation of Modbus/TCP in WIFI Application communication. Inner Mongolia University, Hohhot.

[7] Ma, W.L., Tan, X.G. and Du, W.T. (2017) Multi-Point Power Quality Monitoring in Substations Based on Modbus. Measurement and Control Technology, 36, 115-118.

[8] Shen, Y.F., Bao, J.X. and Zhao, X.W. (2017) Wireless Monitoring System of workshop Environment Based on LabView. Instrumentation Technology and Sensors, 83-87.

[9] Onibonoje, M.O. and Baandele, J.O. (2020) Digimesh-Based Design of a Wireless Monitoring Network for Environmental Factors Affecting Granary System. Interna- 
tional Journal of Engineering Research in Africa, 48, 126-1332.

[10] Jaber, A.S. and Idrees, A.K. (2020) Adaptive Rate Energy-Saving Data Collecting Technique for Health Monitoring in Wireless Body Sensor Networks. International Journal of Communication Systems, 33. 